\title{
Fluorescence Lifetime Imaging Endoscopy
}

\author{
G T Kennedy ${ }^{\mathrm{a}}$, S Coda ${ }^{\mathrm{c}}$, A J Thompson ${ }^{\mathrm{a}}$, D S Elson ${ }^{\mathrm{b}}, \mathrm{M}$ A A Neil ${ }^{\mathrm{a}}$, G W Stamp ${ }^{\mathrm{c}}$, A Thillainayagam ${ }^{\mathrm{d}}$, \\ B Viellerobe $^{\mathrm{e}}, \mathrm{F}$ Lacombe $^{\mathrm{e}}, \mathrm{C}$ Dunsby ${ }^{\mathrm{a}, \mathrm{c}}$, and P M W French ${ }^{\mathrm{a}}$ \\ ${ }^{a}$ Department of Physics, Imperial College London, South Kensington Campus, London, SW7 2AZ, \\ UK \\ ${ }^{\mathrm{b}}$ Department of Surgery and Cancer, Faculty of Medicine, Imperial College London, South \\ Kensington Campus, London, SW7 2AZ, UK \\ ${ }^{c}$ Division of Experimental Medicine, Faculty of Medicine, Imperial College London, Hammersmith \\ Campus, London, W12 0NN, UK \\ ${ }^{\mathrm{d}}$ Department of Gastroenterology, Faculty of Medicine, Imperial College London, Hammersmith \\ Campus, London, W12 0NN, UK \\ ${ }^{\mathrm{e}}$ Mauna Kea Technologies, 9 rue d'Enghien - 75010 Paris, France
}

\begin{abstract}
We present two FLIM endoscopes for clinical imaging and in vivo cell biology. For subcellular confocal imaging we demonstrated the first confocal FLIM endomicroscope, implementing TCSPC with a Cellvizio ${ }^{\circledR}$ GI, which we have now developed as a self-contained wheeled instrument $(1.0 \times 0.7 \mathrm{~m})$ incorporating a tunable excitation laser and acquiring images in $<10 \mathrm{~s}$. This has been applied to image FRET in live cells and to image tissue autofluorescence, for which we are implementing "FIFO" for image montaging. For diagnostic screening/guided biopsy, we have developed a complementary wide-field FLIM endoscope employing time-gated detection with violet and UV excitation for imaging over mm-cm fields of view.
\end{abstract}

Keywords: Endoscopic imaging; Confocal microscopy; Fluorescence microscopy; Scanning microscopy.

\section{INTRODUCTION}

Optical fibre confocal microscopes and endomicroscopes [1-13] can provide optically sectioned cellular imaging in vivo with a resolution similar to conventional histopathology. This ability to perform optical biopsies in situ could assist in the early identification of cancerous and precancerous tissue by informing and guiding conventional biopsy. Fluorescence lifetime imaging can provide molecular contrast and report on the local molecular environment and has been shown to provide label-free contrast between normal and diseased tissue, e.g. [8, 14-16]. It is already widely used to read out fluorescence sensors [17] and Förster resonant energy transfer (FRET) to probe molecular interactions [18]. Furthermore, since FLIM is an inherently ratiometric technique it is less prone to intensity artefacts associated with factors such as fluorophore concentration, non-uniform illumination, tissue deformation and scattering.

To this end a range of endoscopes have been demonstrated previously using a variety of imaging methods such as reflected light [5,9], single photon excited $[1-4,6-8,12,13]$ and multiphoton excited $[10,11]$ fluorescence, multispectral $[3,4]$ and fluorescence lifetime $[7,8,12,13]$ imaging in both coherent fibre bundle $[1-6,12,13]$ and scanning single fibre [7-10] geometries. In this paper, we present our recent progress in developing clinically deployable FLIM endoscopy instrumentation. 


\section{CONFOCAL FLUORESCENCE LIFETIME IMAGING ENDOMICROSCOPE}

The optical configuration of the confocal FLIM endomicroscope is described in detail in Reference 12. Briefly, the system was based on a commercially available and clinically deployed laser scanning endomicroscope [6] (Cellvizio ${ }^{\circledR}$ GI, Mauna Kea Technologies). This instrument is a laser scanning single-photon fluorescence fibre bundle endomicroscope capable of recording optically sectioned images at 12 frames per second using continuous wave excitation at $488 \mathrm{~nm}$ wavelength with a variety of fibre-optic probes. For our experiments we used a coherent bundle consisting of 30,000 fibers terminated with a miniature objective (Cellvizio ${ }^{\circledR}$ Mini O). This probe provides a transverse resolution of $\sim 1.4 \mu \mathrm{m}$, a working distance of $60 \mu \mathrm{m}$ and a field of view of $240 \mu \mathrm{m}$. The diameter of the distal tip is $2.6 \mathrm{~mm}$, which allows it to be passed down the working channels of endoscopes

For FLIM, pulses from a fibre-coupled modelocked frequency-doubled Ti:Sapphire laser operating at a repetition rate of $80 \mathrm{MHz}$ were coupled into the internal beam path of the endoscope immediately before the scanning assembly. This external beam could be readily switched with the internal laser beam to enable interchanging between FLIM and the standard fluorescence imaging endoscope configuration (i.e. using the internal, filters, detector and software acquisition). Fluorescence from the sample was collected by the fiber probe, filtered from the excitation light using a dichroic beamsplitter and emission filter, and then focused through a $15 \mu \mathrm{m}$ diameter pinhole onto a photomultiplier. FLIM data was acquired using a commercial time correlated single photon counting (TCSPC) card (SPC830, Becker and Hickl, $\mathrm{GmbH}$ ) where the line and frame synchronization signals were obtained from the endoscope electronics and the pixel clock was derived from the TSCPC card. A schematic of the system is presented in Fig.1, together with a photograph of the system assembled on a trolley having a footprint of $1.0 \times 0.7 \mathrm{~m}$ for deployment in the endoscopy suite.
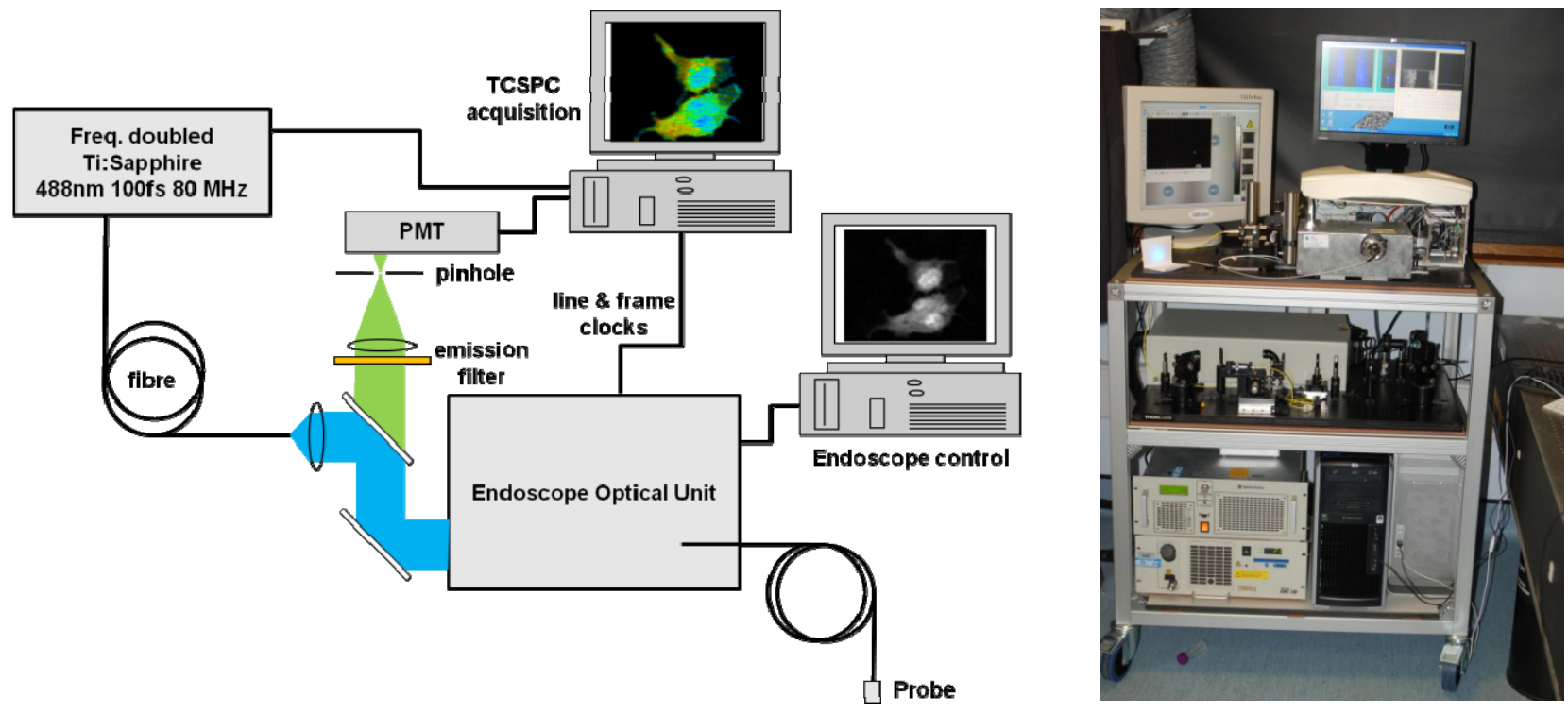

Figure 1. Schematic of the confocal FLIM endomicroscope (left) and photograph of the trolley mounted system (right). Adapted from [12] and [13].

As a first step towards clinical application to biological tissue autofluorescence, the FLIM confocal endomicroscope was applied to unstained cross sections of rat tail tendon. Figure 2 shows the fluorescence intensity and lifetime images obtained, together with a transmission micrograph of an adjacent H\&E stained section. 

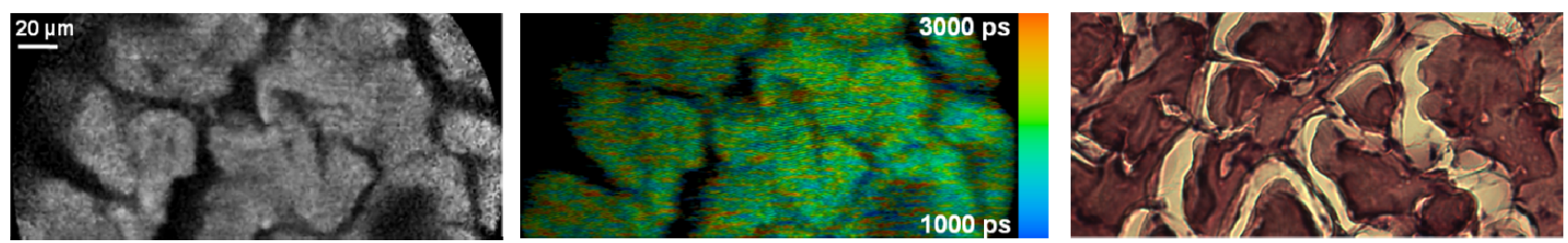

Figure 2. Tat tail tendon. Intensity image (left), Merged fluorescence lifetime image (center) and transmission micrograph of parallel $\mathrm{H} \& \mathrm{E}$ stained section. Adapted from [12].

The confocal FLIM endomicroscope was also applied image Förster resonant energy transfer (FRET) in live cells [13]. In this work we imaged live COS-7 cells that had been transfected with either green fluorescent protein (eGFP) or a tandem construct consisting of eGFP directly linked to the fluorescent protein mCherry. For these experiments the excitation wavelength was $481 \mathrm{~nm}$ and an excitation filter was used to select the emission from the eGFP only. Figure 3 shows the FLIM images obtained for the cells, together with their lifetime histograms. The cells transfected with the tandem construct exhibit shorter lifetimes, clearly indicating the presence of FRET. Full resolution images $(350 \times 512$ pixels) required $60 \mathrm{~s}$ acquisition time for an average incident power of $360 \mu \mathrm{W}$ and the detected count rate was $3.5 \times 10^{5}$ $\mathrm{cs}^{-1}$. However, by binning the pixels in the analysis software, it was possible to reduce the acquisition to $1 \mathrm{~s}$, albeit with some loss of resolution as shown in figure 3.
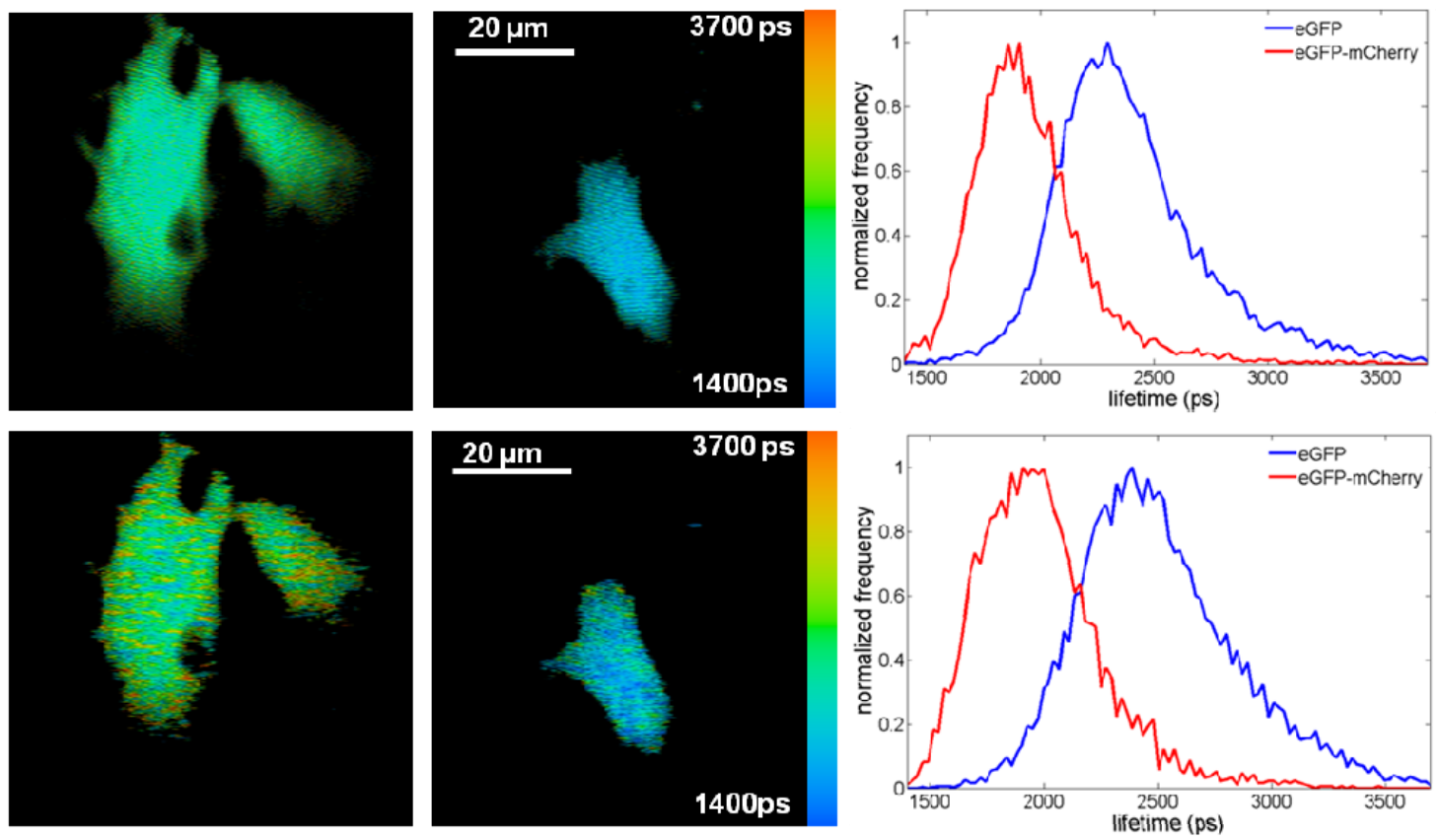

Figure 3. FLIM images of COS cells transfected with only eGFP (left), eGFP linked to mCherry (middle) and histogram of the pixel lifetimes (right). Top row: 60s acquisition duration, 1x1 pixel binning. Bottom row: 1s acquisition, $5 \times 5$ pixel binning.

\subsection{FIFO data processing and image montaging}

In our previous work the FLIM data was acquired using the histogram mode of the TCSPC card. In this mode of operation, the histogram of the photon arrival times is generated and stored in the on-board memory of the TCSPC card before being transferred to the host computer at the end of the acquisition. The $32 \mathrm{MB}$ onboard memory limited the image size to $350 \times 512$ pixels with 64 time bins, which was not sufficient to cover the entire field of view of the fibre 
probe. Furthermore, the time required to save the data to disc and subsequently process it into fluorescence lifetime images was significant, preventing viewing of the lifetime data during imaging. To address these issues we operated the TCSPC card in first-in first-out (FIFO) acquisition mode. In this configuration the events corresponding to line clocks, frame clocks and detected photons were transferred to the host computer and in-house software was then used to process the data into fluorescence lifetime images. This allows much larger image sizes, greater sampling of the fiber bundle and finer photon arrival time resolution, if required. Importantly, it also allows the rapid calculation of the mean photon arrival time that can be quickly processed into fluorescence lifetime or intensity-merged fluorescence lifetime images which could be viewed in "real time" during imaging. Since the field of view of the fibre probe is relatively small (250 $600 \mu \mathrm{m})$ it is desirable to be able to view larger fields by montaging successive images as the fibre is moved across the sample. As an initial demonstration we have implemented a simple model based on edge detection and cross correlation of images acquired in consecutive time windows. This allows the relative shift between images to be determined and for them to be stitched together in software to achieve a larger field of view.

\section{WIDE FIELD ENDOSCOPE}

Whilst the confocal FLIM endomicroscope described above gives high-resolution optically-sectioned images with a spatial resolution similar to conventional histopathology, it is often desirable to rapidly image much larger fields of view than is possible with such laser scanning instruments, e.g. for "red flag" guided biopsy screening and applications that can tolerate lower transverse and axial resolutions. To this end we are developing a handheld wide-field FLIM endoscope based on time gated imaging using a time-gated image intensifier.

For our first implementation, we imaged the output of a rigid clinical arthroscope onto a custom-built gated optical intensifier (modified HRI, Kentech Instruments Ltd). The body of the HRI was modified by the manufacturer to reduce its size and to provide a handgrip. The phosphor of the gated optical intensifier was relayed onto an industrial grade CCD camera using a fiber taper and a photograph of the system is shown in Figure 4. We note that the system is not restricted to using a rigid arthroscope and will accept any standard endoscope eyepiece. Fluorescence excitation light was provided either by a fiber-coupled modelocked frequency-doubled Ti:Sapphire laser, tunable from $360-495 \mathrm{~nm}$, or by a compact UV fiber laser at $355 \mathrm{~nm}$.
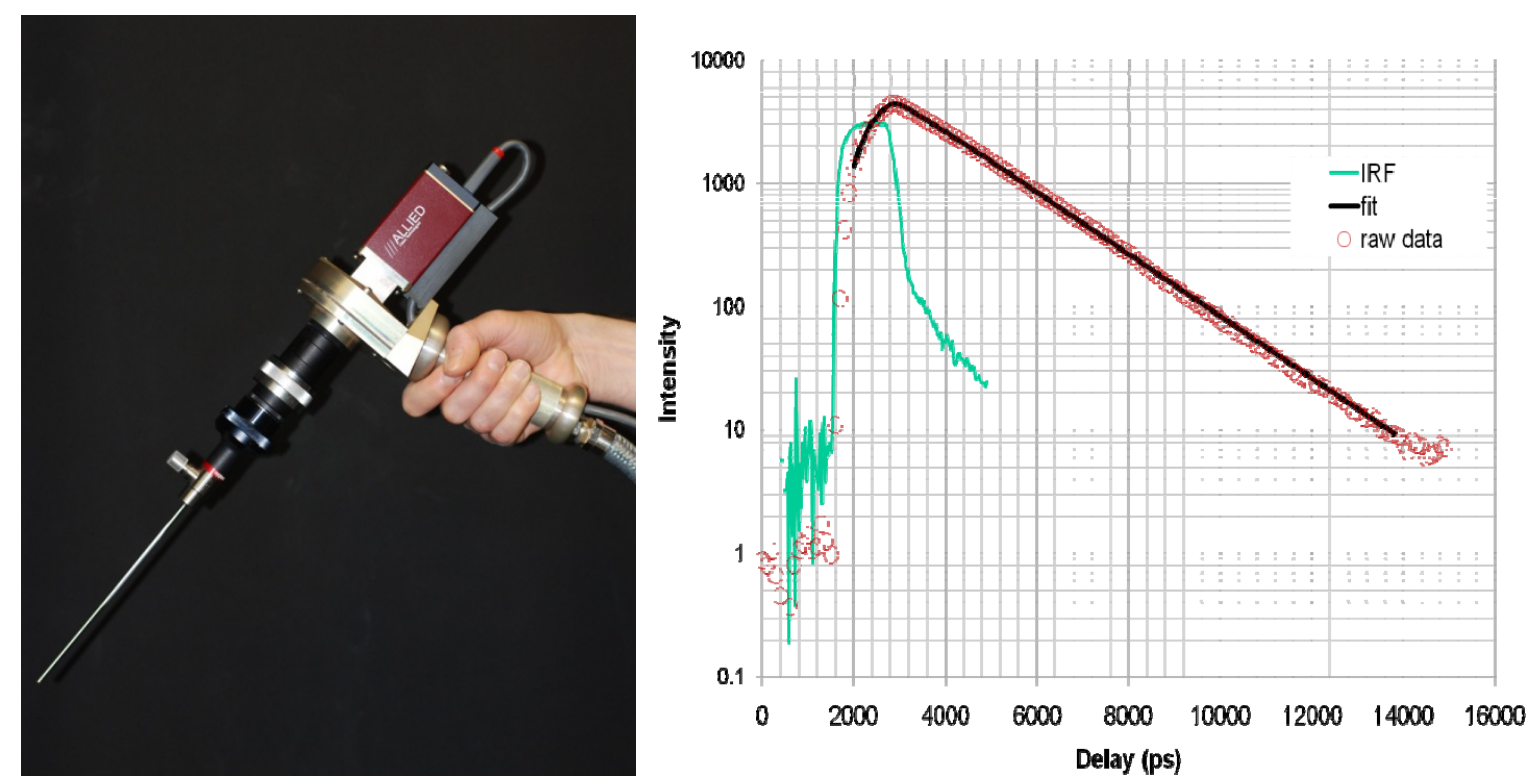

Figure 4. Photograph of the hand-held widefield FLIM endoscope employing a rigid artheroscope (left) and verification of performance using a solution rhodamine $\mathrm{B}$ as a single-exponential lifetime standard 
This FLIM endoscope was first tested on a solution of Rhodamine B that is known to have a single exponential fluorescence decay. The fitted curve yielded a lifetime of $1.73 \mathrm{~ns}$, in agreement with published data [19], see figure 4. Subsequently a "tru-cut" biopsy of normal was bladder tissue was imaged using $405 \mathrm{~nm}$ excitation light with a $430 \mathrm{~nm}$ long pass emission filter. Six $1 \mathrm{~ns}$ time gated images were acquired with a $0.2 \mathrm{~s}$ exposure per gate. The resulting FLIM map and fluorescence lifetime histogram are presented as Figure 5. These initial measurements are in good agreement with the results obtained from a previous wide-field system, although further work needs to be carried out to thoroughly characterize the endoscope.
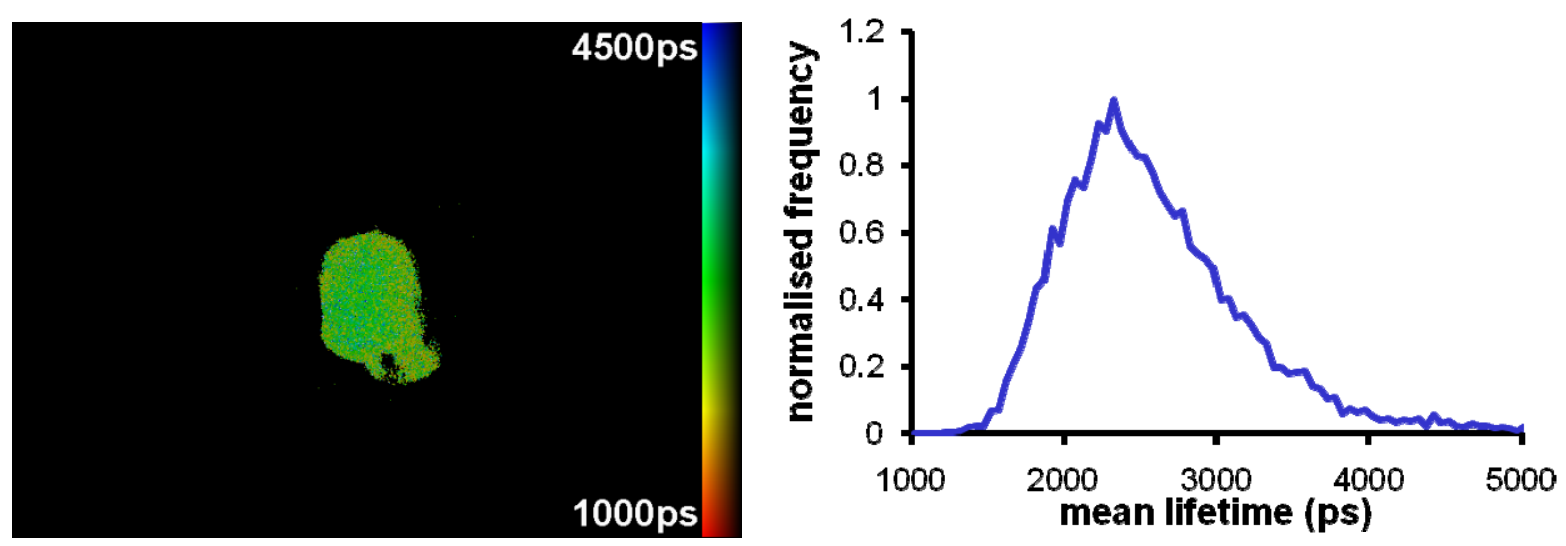

Figure 5. FLIM image obtained with the handheld rigid FLIM endoscope shown in figure 4. The sample was a tru-cut biopsy of normal human bladder tissue.

\section{CONCLUSIONS}

We have presented details of FLIM endoscopes designed for clinical applications and to study molecular cell biology in live disease models. For endoscopic FLIM with subcellular resolution, we described a laser scanning confocal FLIM endomicroscope incorporating time correlated single photon counting (TSCPC) that enables optically sectioned endoscopic FLIM of fields of view up to $600 \mu \mathrm{m}$ via fiber-optic bundle probes. For clinical applications we have now developed a self-contained wheeled instrument, of $1.0 \times 0.7 \mathrm{~m}$ footprint, that incorporates a tunable ultrafast excitation laser and all electronics. This endoscope has been applied to FRET in cells expressing GFP-based fluorophores and to tissue autofluorescence for label-free imaging of tissue. We believe it is appropriate for in vivo optical biopsy applications for which we have implemented "FIFO" FLIM for live imaging and image montaging. For diagnostic screening and image guided biopsy "red flag" applications, a larger field of view is often desirable and to this end we have developed a complementary wide-field hand-held FLIM endoscope employing a compact gated optical intensifier. This endoscope enables rapid wide-field (non-sectioned) FLIM of tissue autofluorescence with fields of view up to several $\mathrm{cm}$.

\section{ACKNOWLEDGEMENTS}

This work was supported by grants from the Engineering and Physical Science Research Council (EPSRC): Endoscopic FLIM for label-free tissue contrast (EP/F040202/1). We are also grateful to Kentech Instruments Ltd for their support. Paul French acknowledges a Royal Society Wolfson Research Merit Award. 


\section{REFERENCES}

[1] Harris, M. "Fibre bundle laser confocal scanning microscope." PCT/AU/00129, WO91/15792. (1991).

[2] Gmitro, A. F. and Aziz, D. "Confocal microscopy through a fiberoptic imaging bundle," Optics Letters 18, 565567 (1993).

[3] Rouse, A. R. and Gmitro, A. F. "Multispectral imaging with a confocal microendoscope," Optics Letters 25, 1708-1710 (2000).

[4] Jean, F., Bourg-Heckly G. and Viellerobe B., "Fibered confocal spectroscopy and multicolor imaging system for in vivo fluorescence analysis," Optics Express 15, 4008-40017 (2007).

[5] Liang, C., Descour, M. R., Sung, K. B., and Richards-Kortum, R.,"Fiber confocal reflectance microscope (FCRM) for in-vivo imaging," Optics Express 9, 821-830 (2001).

[6] Laemmel, E., Genet, M., Le Goualher, G., Perchant, A., Le Gargasson, J. F., and Vicaut, E., "Fibered confocal fluorescence microscopy (Cell-viZio (TM)) facilitates extended imaging in the field of microcirculation - A comparison with intravital microscopy," Journal of Vascular Research 41, 400-411 (2004).

[7] Ghiggino, K. P., Harris, M. R., and. Spizzirri, P. G, "Fluorescence lifetime measurements using a novel fiberoptic laser scanning confocal microscope," Review of Scientific Instruments 63, 2999-3002 (1992).

[8] Wagnières, G., Mizeret, J., Studzinski, A., and van den Bergh, H., "Frequency-domain fluorescence lifetime imaging for endoscopic clinical cancer photodetection: Apparatus design and preliminary results," Journal of Fluorescence 7, 75 (1997).

[9] Dickensheets, D. L. and Kino, G. S., "Silicon-micromachined scanning confocal optical microscope," J. Microelectromech. Syst. 7, 38-47 (1998).

[10]Helmchen, F., Fee, M. S., Tank, D. W. and Denk, W., "A miniature head-mounted two-photon microscope: High-resolution brain imaging in freely moving animals," Neuron 31, 903-912 (2001).

[11]Lelek, M., Suran, E., Louradour, F., Barthelemy, A., Viellerobe, B. and Lacombe, F., "Coherent femtosecond pulse shaping for the optimization of a non-linear micro-endoscope,"Opt. Express 15, 10154-10162 (2007).

[12] Kennedy, G. T., Manning, H. B., Elson, D. S., Neil, M. A. A., Stamp, G. W., Viellerobe, B., Lacombe, F., Dunsby; C. and French, P. M. W., "A fluorescence lifetime imaging scanning confocal endomicroscope" J. Biophoton.3, 103-107 (2010).

[13] Kumar, S., Alibhai, D., Margineanu, A., Laine, R., Kennedy, G., McGinty, J., Warren, S., Alexandrov, Y., Munro, I., Talbot, C., Stuckey, D. W., Kimberly, C., Viellerobe, B., Lacombe, F., Lam, E. W.-F., Taylor, H., Dallman, M. J., Stamp, G., Murray, E. J., Stuhmeier, F., Sardini, A., Katan, M., Elson D. S., Neil, M. A. A., Dunsby, C. and French, P. M. W., "FLIM FRET Technology for Drug Discovery: Automated Multiwell-Plate High-Content Analysis, Multiplexed Readouts and Application in Situ," ChemPhysChem 12, 627-633 (2011).

[14] Munro, I., McGinty, J., Galletly, N., Requejo-Isidro, J., Lanigan, P. M. P., Elson, D. S., Dunsby, C., Neil, M. A. A., Lever, M. J., Stamp, G. W. H. and French, P. M. W., "Towards the clinical application of time-domain fluorescence lifetime imaging," J Biomed Opt 10, 051403 (2005).

[15]Elson, D. S., Galletly, N., Talbot, C., Requejo-Isidro, J., McGinty, J., Dunsby, C., Lanigan, P. M. P., Munro, I., Benninger, R. K. P., de Beule, P., Auksorius, E., Hegyi, L., Sandison, A., Wallace, A., Soutter, P., Neil, M. A. A., Lever, J., Stamp, G. W. and French, P. M. W., [Reviews in Fluorescence], Springer, 1. (2006)

[16] Skala, M. C., Riching, K. M., Bird, D. K., Gendron-Fitzpatrick, A., Eickhoff, J., Eliceiri, K. W., Keely, P. J. and Ramanujam, N., "In vivo multiphoton fluorescence lifetime imaging of protein-bound and free nicotinamide adenine dinucleotide in normal and precancerous epithelia," J Biomed Opt 12, 024014 (2007).

[17] Agronskaia, A. V., Tertoolen, L. and Gerritsen, H. C., "High frame rate fluorescence lifetime imaging," J Phys D-Appl Phys 36, 1655-1662 (2003).

[18] Wouters F. S. and Bastiaens, P. I. H., "Fluorescence lifetime imaging of receptor tyrosine kinase activity in cells," Curr Biol 9, 1127-1130 (1999).

[19] Boens, N., Qin, W. W., Basaric, N., Hofkens, J., Ameloot, M., Pouget, J., Lefevre, J. P., Valeur, B., Gratton, E., Vandeven, M., Silva, N. D., Engelborghs, Y., Willaert, K., Sillen, A., Rumbles, G., Phillips, D., Visser, A., van Hoek, A., Lakowicz, J. R., Malak, H., Gryczynski, I., Szabo A. G., Krajcarski, D. T., Tamai, N. and Miura, A., "Fluorescence Lifetime Standards for Time and Frequency Domain Fluorescence Spectroscopy," Anal Chem 79, 2137-2149 (2007). 